\title{
Poétesses en marge
}

Un cas d'interdiction de la parole poétique féminine

\section{Amalia Dragani}

\section{(2) OpenEdition}

Journals

Édition électronique

URL : https://journals.openedition.org/clo/2345

DOI : $10.4000 /$ clo.2345

ISSN : 2266-1816

Éditeur

INALCO

Édition imprimée

Date de publication : 31 décembre 2015

ISBN : 9782858312276

ISSN : 0396-891X

Référence électronique

Amalia Dragani, «Poétesses en marge », Cahiers de littérature orale [En ligne], 77-78 | 2015, mis en ligne le 10 mai 2016, consulté le 30 juin 2021. URL : http://journals.openedition.org/clo/2345 ; DOI : https:// doi.org/10.4000/clo.2345

Cahiers de littérature orale est mis à disposition selon les termes de la Licence Creative Commons Attribution - Pas d'Utilisation Commerciale 4.0 International. 


\title{
Poétesses en marge Un cas d'interdiction de la parole poétique féminine
}

\author{
Amalia Dragani \\ Las, EHESS, Paris Sciences et Lettres \\ IIAC-Lahic \\ $\hat{E}$ Tăkaribt, serbo e dd-ikka \\ Oh Tăkaribt, l'honneur reviendra ${ }^{1}$
}

À partir d'un cas ethnographique - un concours de poésie touarègue au sein d'un festival au nord du Niger - j'aborderai la relation entre l'espace public et religieux, le genre et les normes, contestés, d'usage de la parole à la veille de la rébellion touarègue qui fut déclenchée le 7 février 2007, par l'attaque-surprise de la caserne militaire d'Iférouane, où avait lieu le festival ${ }^{2}$.

L'exclusion des femmes du concours poétique suscita différentes réactions de la part des intéressées, en fonction de leur séniorité. Les poétesses les plus jeunes, autrices de vers célèbres pendant la rébellion des années 1990, furent marginalisées en raison du contenu politique de leur production. Dans un état d'aliénation symbolique, non autorisées à parler, mais restant visibles et audibles par tous, elles se produisirent sur la scène du festival en claquant rythmiquement des mains et en émettant des youyou.

1. Charles de Foucauld, 1925-1930, p. 36.

2. Je remercie sincèrement Dominique Casajus pour ses précieux commentaires ; Alexandre Dauphin pour la première relecture ; Adouma agg Alghoubas, Moussa agg Elekou et Mohamed agg Ahmedou pour notre effort collectif de traduction de ces poèmes, que j'ai enregistrés en 2006 au Niger. 
Une poétesse seulement, T., tirant légitimité de son aînesse, s'autorisa à saboter le concours, en s'invitant à tout moment sur la scène, en volant le microphone et en déclamant ses tišiway (poèmes). Si pour le présentateur, qui tentait de dissimuler les tensions en la disqualifiant, elle n'était qu'une « folle qui n'en faisait qu’à sa tête », pour les poètes, bien au contraire, elle représentait « une vraie tamajaq », c'est-à-dire une Touarègue de noble naissance, qui n'acceptait pas de se soumettre à un ordre exogène qu'elle n'avait pas contribué à créer.

Le geste de T. contre un dispositif répressif, situé en rupture historique avec la conception touarègue du genre et de l'accès féminin au pouvoir, n'est pas aujourd'hui sans rappeler les manifestations de femmes touarègues à Kidal contre les islamistes, face aux atteintes à leurs libertés.

L'Islam réformateur se répandait au Niger à l'époque du festival et les prêches avaient pour cible les repères identitaires nomades tels que les soirées musicales et poétiques caractérisées par la mixité sexuelle, la consommation de boissons réputées excitantes comme le thé, l'écriture « profane » touarègue (utilisant les caractères de l'alphabet local, les tifinagh) et la pratique de la poésie et de la musique.

L'exhibition publique de la voix féminine, considérée comme séduisante et diabolique, était non seulement de plus en plus désapprouvée, mais même perçue comme un signe de débauche et reléguée en dehors de l'espace public.

\title{
$\breve{A z z a ̆ m a n ~(l ' e ́ p o q u e), ~ u n e ~ o a s i s ~ a u ~ c a r r e f o u r ~ d e ~ t o u s ~ l e s ~ e ́ c h a n g e s ~}$
}

\author{
Tehoulem-in Tăkaribt, lejouad \\ Saluez de ma part Tăkaribt, hommes nobles!

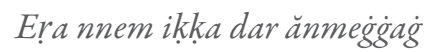 \\ Tu as été vengée en un pays étranger ${ }^{3}$.
}

Alors que les Touaregs appellent l'époque présente ăzzăman (ce qui veut dire le « temps », la « période »), le même terme peut signifier également une « époque de guerre », « le début des hostilités ». Ce mot qui désigne la contemporanéité est à l'origine de l'expression Kel Ăzzăman, « ceux de l'époque » pour désigner les Touaregs contemporains et se sentant tenus de défendre, par les armes, leur spécificité culturelle.

L'époque dont je parlerai dans cet article coïncide avec la veille de la rébellion déclenchée par le mouvement rebelle MNJ (Mouvement des Nigériens pour la Justice) entre 2007 et 2009. Durant mon terrain (entre 2004 et 2007), le nord du

3. Charles De Foucauld, 1925-1930, p. 124. 
Niger venait de sortir d'une longue guerre civile et était confronté non seulement à l'après-conflit, mais aussi à des transformations complexes au niveau institutionnel. Si le mot « démocratie » (demokrasi) se prêtait à l'un des nombreux jeux de mots desquels les Touaregs sont friands (Bernus, 1989) du fait de sa proximité sonore avec le terme demokarasi (vol), le mot laik (laïque) était, lui, utilisé dans le langage courant comme un synonyme de « corrompu ».

La libéralisation politique amorcée depuis le début des années 1990 avait enfanté les partis politiques (en tamacheq fartităn), implantés dans les villages avec des leaders plus ou moins importants en fonction des ressources économiques et symboliques qu'ils pouvaient mobiliser. Les communes (kominaten) ont également fait leur apparition, avec à la tête de chacune un maire (mer) et un conseil communal (konsay), ce qui a introduit un changement non seulement au niveau politique mais aussi dans la perception du territoire.

L'Aïr était parcouru à la fois par les migrants en provenance de l'Afrique subsaharienne et les caravaniers de la taghlamt (la caravane de sel) qui partent encore chaque année et traversent le désert du Ténéré pour échanger le mil contre du sel dans les oasis toubous de Bilma et Fachi. Les voitures de tourisme (taksităn) croisaient celles des trafiquants de frod (fraude) à la frontière libyco-nigérienne.

La capacité d'attraction de l'Aïr était à son apogée à cette époque, ce qui faisait de la région d'Agadez un site surinvesti par les « Occidentaux » : touristes, membres des ONG et des organismes internationaux, chercheurs dits inassafika et agents des services secrets (răffototăn), journalistes ou kel daga (« ceux de l'éloquence ») et réalisateurs à la recherche du « mythe touareg » ${ }^{4}$. Leur présence n'était pas sans conséquence sur mon travail de recherche qui devait se confronter à des informateurs particulièrement sollicités et rémunérés.

En outre, les Touaregs représentaient une population sur-enquêtée par plusieurs générations d'anthropologues, pour me différencier desquels j'ai dû recourir à des « hors-pistes $»^{5}$, des ajustements méthodologiques basés sur une approche ethnobiographique (Fabre et al., 2010), qui m'ont permis, en m'intéressant aux poètes davantage qu'à leurs « textes » oraux, de mieux percevoir les coulisses du festival et les tensions qui entouraient le concours de poésie.

L'Aïr du début du siècle était perçu par les Touaregs comme un lieu contenant à profusion d'albaraka (bénédiction divine) et parsemé de lieux sacrés (al hima) dispensateurs de cette force. Aujourd'hui, beaucoup de Touaregs expriment une volonté de se protéger des étrangers, perçus comme des intrus agressifs et

\footnotetext{
4. Paul Pandolfi, 2001.

5. Anne Doquet, 2007.
} 
irrespectueux de leurs traditions. Ils manifestent leur ambivalence en élaborant mentalement des rapports entre leurs intrusions et d'éventuels phénomènes malheureux survenus avant, après ou pendant leur séjour : par exemple, la contamination du VHS/SIDA était associée aux rapports non protégés avec les touristes et les prostituées béninoises qui cherchaient à rejoindre l'Europe via l'Aïr.

La préoccupation liée à la préservation des lieux inclut un contrôle accru du corps féminin (Rasmussen, 2004a ; 2004b) comme réponse à l'intrusion des étrangers dans les lieux sacrés et au danger constitué par un abus du pouvoir de l'albaraka de la part des marabouts corrompus par les temps. Si la stérilité était autrefois imputée, dans la société touarègue, tant à l'homme qu'à la femme, aujourd'hui elle est de plus en plus attribuée aux femmes, en particulier au sein des communautés sédentarisées. Les femmes sont de plus en plus jugées responsables d'effets négatifs sur leur mari, principalement chez les Touaregs agropasteurs et sédentaires où elles sont accusées d'éloigner l'albaraka des terres de leur époux ; cette accusation a été la cause de nombreux divorces.

Il est nécessaire de préciser que cette région du monde touareg est singulière par rapport aux autres : les relations étroites avec les populations haoussaphones du sud, parmi lesquelles les caravaniers vivaient autrefois à peu près six mois par an lors de leur séjour dans la région de Kano (actuel nord du Nigeria), sont à l'origine de l'introduction ancienne de la polygynie, inusuelle chez la plupart des groupes touaregs ; notamment la bigamie ou le concubinage institutionnalisé avec une taklit, femme de condition servile, d'où le métissage plus important qu'ailleurs.

Une montée en puissance de l'Islam rigoriste dans la région et dans la sphère publique se manifestait par des expressions de contrôle sur les habitudes alimentaires, sexuelles et culturelles (attaques contre les caractères tifinagh, alphabet profane considéré comme inauthentique par rapport à l'arabe du Coran, mais aussi contre la poésie trop centrée sur des thématiques amoureuses, ou contre la mixité sexuelle lors de soirées). La circulation d'images de Ben Laden (trois ans après le 11 septembre 2001), affichées aux murs ou sur des porte-clés était peu répandue mais visible sur les marchés et dans les chambres des célibataires.

Considérée de plus en plus comme intrinsèquement érotique à cause de son association avec le corps auquel elle renvoie (Tauzin, 2007), la voix féminine était tenue responsable de l'《 adultère des oreilles ».

Le statut élevé de la femme dans la société touarègue ne pouvait qu'attirer les foudres des prêcheurs rigoristes. Ce statut s'exprime entre autres par une liberté sexuelle accordée aux jeunes filles (asri), aux femmes divorcées ou aux veuves, et le droit de choisir leur mari (surtout après le premier divorce), par la participation des femmes aux activités publiques (Claudot-Hawad, 1993), l'absence de ségrégation, 
l'intégration de facto des enfants illégitimes par le groupe maternel, la possibilité pour les hommes et les femmes de danser ensemble, l'utilisation parfois des matronymes ainsi que la reconnaissance de la position sociale du frère de la mère.

\section{Le festival de l'Aïr : pratiques protocolaires}

Tehoulem-in Tăkaribt dimzâd Saluez Tăkaribt et les joueuses de violon (litt. le violon) ${ }^{6}$.

J'ai assisté au festival de l'Aïr à deux reprises, du 27 au 29 décembre 2005 et du 26 au 29 décembre 2006, à Iférouane, une oasis située dans la région montagneuse de l'Aïr oriental, à $320 \mathrm{~km}$ au nord de l'ancien centre caravanier d'Agadez.

Au carrefour de plusieurs hameaux et campements, Iférouane constitue une zone de maraîchage et d'élevage, bordée à l'est par le désert du Ténéré et à l'ouest par la plaine argileuse de Talak où se trouve la ville de Gougaram. Dans ce paysage cristallin et montagneux, recouvert d'une patine noirâtre et luisante, de vastes étendues ocres, des plateaux rocailleux, dans lesquels s'encaisse le réseau des koris (lits de rivières souvent à sec), des dômes en forme de pain de sucre, des crêtes, des blocs et des boules, des cônes volcaniques et des coulées de lave basaltiques se succèdent.

Dans une tendance à la « permanentisation $\gg^{7}$ des festivals et dans une logique de « reproduction à l'identique $»^{8}$, Iférouane fut le site privilégié des six premières éditions, avant le déclenchement de la rébellion de 2007-2009.

Créé en 2001, à la suite d'une série de foires artisanales, il regroupe de nombreuses communautés de l'Aïr, les maires et les responsables des projets de développement, ainsi que de nombreuses localités avoisinantes y prennent part : Tadeck, Tin Telloust, Tabelot, Timia, Iférouane, Gougaram, Aoudéras, Dabaga, Elmeki et Arlit.

Le festival s'articulait sur trois jours et différents concours y figuraient, entre autres : le concours de port du turban, de tresses féminines, de tenues traditionnelles masculines et féminines, les concours de chameaux (le mieux dressé, le plus beau, le mieux harnaché), d'ânes (le mieux harnaché) et une course finale de chameaux, de danses masculines et de chants de mariage et de fête.

6. Charles de Foucauld, 1925-1930, p. 124.

7. Mahalia Lassibille, 2006.

8. Arjun Appadurai, 2001. 
Malgré son projet de requalification des traditions immatérielles locales dans le cadre de la décentralisation, le festival était marqué symboliquement par la visibilité de l'État (addawlat en tamacheq) : la présence du drapeau nigérien et des autorités représentatives des institutions nationales (hommes politiques, assayasăten), et l'emploi de la langue française par le présentateur haoussaphone.

Le protocole suivi lors du festival renforce les valeurs, croyances et conventions légitimant l'autorité politique. De multiples querelles symboliques ont pour objet le rang assigné, la place occupée et la position assise ou debout, la hauteur du siège attribué, la proximité ou la distance de l'autorité, l'ordre d'entrée et de déplacement. Le protocole est un ordre, un mode de répartition des corps, un dispositif contribuant à mettre en scène le pouvoir politique. L'agencement particulier du lieu du festival (où, au centre, se dresse la loge des autorités, inaccessible au commun des mortels) introduisait une rupture symbolique avec les performances traditionnelles, rapprochant cet espace des meetings électoraux, où l'État et les partis politiques ( fartităn) se mettent en scène. Dans ce festival, les notables, protégés par des gardes armés (sožităn), étaient accueillis dans une tribune d'honneur en banco, qui présentait une arcade de cinq piliers peints aux couleurs de la bannière nigérienne et surmontée d'un long drapeau. Ce dernier se révélait être en réalité un turban masculin et les emblèmes vexillaires nigériens avaient été, pour ainsi dire, à l'occasion du festival, « targuisés », de manière à ce que la bannière nationale coïncide avec le chèche, symbole de l'identité vestimentaire masculine et avec le pilier (tagattăwt) renvoyant, lui, aux piquets de la tente, symbole de l'identité féminine. Au-dessus de la bannière nationale, d'une manière extrêmement significative, une affiche représentant un amajagh, guerrier touareg « aristocrate » de profil sur un chameau blanc, dominait d'en haut l'espace du festival et les membres du goufernman (gouvernement) invités. Le protocole invite à l'obéissance politique qui exige une maîtrise des corps et des postures, permettant de comprendre la « morphologie du pouvoir ».

Devant la loge ombragée, éloigné d'elle par un espace inoccupé, le public, en demi-cercle, formait quatre rangées : au premier rang, des enfants, des mères avec des bébés et des dames assises sur des nattes, une deuxième rangée constituée par des hommes et des femmes debout, suivie par un cordon d'hommes à chameaux. Plus loin encore, sur les pick-up, sur les camions et les $4 \mathrm{X} 4$, des jeunes sont assis ou debout. Ce festival est caractérisé par la mixité sexuelle des rassemblements touaregs où d'autres influences (par exemple arabes en Algérie) ne dominent pas la scène. À la périphérie se trouvaient des hangars et des stands où des cultivateurs vendaient les produits de la région, les fruits de l'oasis de Timia, les fromages de l'Aïr ainsi que des objets d'artisanat et des bijoux.

Dans l'espace utilisé pour le spectacle et proche de la loge, des hommes, des femmes, des enfants et des animaux prennent place. Ils défilent avec leurs objets : 
des violons, différentes sortes de tambours, des radios, des nattes en plastique, des sandales, des parapluies (pour s'abriter du soleil), de l'argent (des pièces mais surtout des billets). En descendant de l'estrade, les notables jettent vers leur artiste préféré une pluie de billets, ils insèrent les billets dans le col du boubou des hommes et aspergent de parfum les femmes.

Au centre de la scène, sont présents les acteurs des professions « modernes »: les cameramen de la RTN (Radiotélévision nigérienne), des journalistes des radios locales (Radio Nomade, Sabara FM, etc.) et de la presse (Aïr Info), des particuliers avec des magnétophones obsolètes, la cassette à bobine à l'intérieur, engagés dans des « patrimonialisations ordinaires ${ }^{9}$.

Toujours debout, microphone à la main, l'animateur haoussaphone du festival occupe un rôle central. Il présente les artistes, amuse le public par des boutades, remercie chaleureusement les notables quand l'un d'eux descend pour jeter de l'argent à profusion, et souligne l'entrée d'une personnalité à peine arrivée sur l'estrade. Dans une configuration spatiale qui témoigne des nouvelles hiérarchies, nul ne prend la parole hormis ceux qui figurent sur la liste des artistes agréés : dans le concours de poésie, les femmes ne sont pas admises à participer.

\section{Renouvellement du code dans l'espace public et conflit dansl'explicitation culturelle, T. contre le protocole}

Alors que la vocation du protocole est de composer un tableau hiérarchique, prévisible et stable, une poétesse tentera de le renverser, appuyée en cela par une partie du public.

T., poétesse (temessheweyt) connue, femme « noble » (tamajaq) et bénéficiant de la dignité que lui confère son âge (taghmart), entre dans l'espace des concurrents et s'assoit à côté des poètes qui déclament. Refoulée, elle surgit à nouveau de la foule, vole le microphone du présentateur, récite ses vers; encore refoulée, elle retourne sur scène, pour en être encore éloignée. Elle n'arrivera à réciter un poème entier qu'une seule fois, poème que nous présenterons dans le dernier paragraphe. À chacune de ses autres tentatives, elle est chassée de la scène. Tandis que le présentateur dit en riant : « voici T., elle est vraiment folle », les poètes ne sont pas du même avis et certains l'incitent et l'encouragent de leurs cris.

L'espace public que constitue la scène était ressenti par les poétesses comme le lieu d'une triple domination dérivant de différentes formes de pouvoir : la domination culturelle occidentale où le masculin est hégémonique dans la sphère

9. Cyril Isnart, 2012. 
publique, la domination de l'État imposant ses hommes et la langue majoritaire, ainsi que les pressions moralisatrices de l'Islam radical.

Si, dans la société traditionnelle, tout le monde sait que la joueuse d'imzad est souvent autant poète que l'homme qui, à ses côtés, récite des vers, on ne peut pas en dire de même pour les Occidentaux (touristes, téléspectateurs) devant lesquels on met en scène l'identité touarègue et ses valeurs culturelles. Le comportement explicite de T. (réciter ses poèmes elle-même, alors que sa présence n'était pas prévue sur scène, contre le protocole) met à nu une fiction culturelle dans le moment où un nouveau code se dessine : le code de l'ăzzăman, de la contemporanéité dans un contexte globalisé.

Or, dans ma thèse et puis dans mon livre (Dragani, 2012), j'ai montré que les femmes de cette région font elles aussi de la poésie mais ne s'exposent pas au public, par pudeur ou par crainte de susciter l'envie (togheršit, le mauvais œil) ou d'attirer les esprits. C'est un récitant (enalbad) ou un autre membre masculin de sa famille qui le fait pour elle, alors que cette dernière joue de l'imzad. Dans l'époque actuelle, se joue le passage de l'implicite à l'explicite, dans un contexte de globalisation et de patrimonialisation, d'injonction à la visibilité et à la prise de parole, en net contraste avec la morale touarègue traditionnelle basée sur l'asshek (la retenue, litt. l'« hésitation ») et la tekarakit (la pudeur).

Les actions de T. apparaissent comme une source de désordre pour les institutions, mais aussi comme un facteur d'unité et de cohésion pour de nombreux Touaregs, aux yeux desquels elle incarne un dépositaire de la tradition. Le seul poème qu'elle réussit à prononcer avant qu'on ne lui retire le microphone rentre parfaitement dans un genre poétique souvent pratiqué par les Touaregs, la satire. Avant d'analyser le poème de T. que j'ai recueilli lors de ce festival, j'illustrerai rapidement les différents genres poétiques avant de me concentrer sur la satire.

\section{Tosawit n-ăbăyak (poésie de critique) et ânargam (épigramme d'invective) chez les poétesses touarègues}

Je présenterai dans un but comparatif des exemples de poèmes féminins anciens, issus des régions montagneuses de l'Ahaggar et de l'Adghagh (aujourd'hui situées à la frontière entre le Mali et l'Algérie).

Ces poèmes ont été collectés par Charles de Foucauld au début $\mathrm{du} \mathrm{Xx}^{\mathrm{e}}$ siècle et sont parus dans un ouvrage posthume en deux volumes (de Foucauld, 19251930). La collecte du Père de Foucauld contient de précieux témoignages, des poèmes féminins recueillis souvent de la bouche même des poétesses. Le fait qu'un homme occidental ait pu collecter des poèmes féminins s'explique par le fait qu'aujourd'hui encore, dans l'ouest du monde touareg et surtout dans la région 
de l'Azawad (actuel Nord du Mali), la poésie féminine n'est pas aussi secrète et son héritage autant « mis en oubli » qu'elle l'est dans les régions orientales (l'Aïr, dont parle cet article, et l'Azawagh, actuel Niger).

En ce qui concerne mon expérience d'ethnologue, en plus des témoignages de mes informateurs, un terrain chez les poètes nomadisant autour des Djebock (Gao, Azawad) m'avait permis de rencontrer sans aucune difficulté des femmes touarègues dont la pratique poétique n'était pas, à ma connaissance, aussi « invisibilisée ».

D'après la classification touarègue des genres littéraires, le nom générique de la poésie est tesawit, qui désigne la poésie en général et comprend à la fois la louange et l'apologie (temmal), la satire (tzssawit n-ăbăyak, litt. « critique ») et l'épigramme d'invective appelée ânargam ainsi que le chant (ašak) et sa variante contemporaine, le chant à la guitare sèche ou électrique (alguitara) rentrée dans les circuits internationaux de la World Music.

Le champ sémantique du rire, du comique et du satirique chez les Touaregs a été l'objet d'un article portant sur les jeux de mots dans la vallée du fleuve fossile Azawagh, au Niger (Bernus, 1989), d'une contribution sur les « paroles à rire » chez les Touregs Kinnin du Tchad (Jay, 1999) et d'un mémoire de maîtrise soutenu à Bamako sur la poésie satirique (Elmeki, 1978).

D'abord, il faudrait rappeler la valorisation de la parole pour un peuple qui se dénomme Kel Tamacheq (litt. « ceux [qui parlent] tamacheq ») ainsi que le pouvoir spécifique qui lui est attribué (Bernus, 1972 ; Casajus, 1987 ; Casajus, 2000).

D'un point de vue linguistique (de Foucauld, 1951 ; Prasse, 2003) la racine du verbe rire $(a d z u)$ est $d z$, d'où, taḍ̆za, le rire, et $a z a ̆ d z o$, la chose qui prête à l'amusement. Les hommes amusants, blagueurs, farceurs et taquins (məzzedzi ou ămazzan) sont très recherchés car ils détendent l'atmosphère avec leur šizăḍaten (plaisanteries).

Le rire est socialement codifié. On peut avoir une relation à plaisanterie avec ses cousins croisés appelés ibubezăn: le verbe « taquiner » (zabbubaz) présente la même racine $b b z$, ce qui exprime le lien très fort entre cousinage croisé et paroles à rires. Une relation à plaisanterie peut avoir lieu, d'après mes interlocuteurs, avec une épouse issue d'un mariage non préférentiel. En schématisant, dans les groupes où le mariage préférentiel se réalise avec une cousine patrilatérale, les relations avec cette dernière seront imprégnées de sérieux tandis qu'avec la cousine matrilatérale une relation à taquinerie sera envisageable. Dans le cas contraire, où le mariage préférentiel se réalise avec la cousine matrilatérale, une relation à plaisanterie sera possible avec la cousine patrilatérale.

Un rire trop intense, d'après C. de Foucauld (1951), se dit hekiki dans le parler septentrional du Hoggar (tahaggart). Cette expression désigne le « rire aux 
éclats » censé attirer les esprits assoiffés toujours à la recherche, dans ces contrées torrides, de sources d'eau pour se désaltérer, telle la salive contenue dans la bouche. Pour se protéger de ces intrus, il est préférable, lorsqu'on rit, de se voiler la bouche pour empêcher l'entrée des diables à l'intérieur du corps, à travers cet orifice. Les Touaregs se couvrent la bouche avec le voile en riant, en pressant un pan de tissu sur la bouche ou se cachent la bouche avec la main. Même rire dans le sommeil est considéré comme un signe néfaste ou, en général, afficher son bonheur en public est réputé dangereux car cela attire l'envie et le « mauvais œil ». Il est préférable de se limiter au sourire et non pas au rire tonitruant.

Ce caractère ambigu du rire est exprimé par le verbe azzan qui signifie plaisanter avec excès, avec une folle gaieté et, en même temps, désigne aussi un railleur insultant en recouvrant le champ sémantique de l'anarchie et du chaos. Par exemple, le mot băqqăbos désigne la compagnie joyeuse, mais aussi le désordre politique et moral, le caractère difficile d'une situation ou imprévisible d'une personne.

S'il ne s'agit pas directement d'injure, puisque le contenu est camouflé et l'agressivité mitigée par le biais de l'ironie, le «poème de critique » (abayak) peut parfois être perçu comme diffamatoire, parce que la cible est présentée de façon péjorative et son image « salie ». Il s'agit d'une sorte de dénonciation publique consistant à dépeindre telle ou telle personne de façon caricaturale et particulièrement agressive.

Des conduites blâmables sont associées aux «dangers » de la parole, comme la taquinerie excessive, la grossièreté, l'euphémisation, la fausse promesse et le faux serment, la médisance, la délation, la divulgation du secret, l'imprécation, la moquerie, la dépréciation des aliments.

L'anthologie du Père de Foucauld (1925-1930) nous donne des exemples de poèmes caricaturaux et satiriques, composés par des poétesses sur des thématiques variées, et aussi des poèmes didactiques, des conseils d'amour par exemple, et bien des critiques et des échanges d'insultes ainsi que de sarcastiques duels verbaux.

Nous allons présenter ici quelques-uns de ces poèmes, dus à des poétesses. Celles-ci composent sur toutes les thématiques de la poésie touarègue : de la guerre à l'amour, de la sécheresse à la religion jusqu'aux anecdotes savoureuses de la vie quotidienne.

Un court poème, ouvre de la poétesse Lalla oult Illi (Lalla fille d'Illi) des Kel Adghagh, née d'après C. de Foucauld en 1870, permettra au lecteur de pénétrer à l'intérieur d'une relation matrimoniale. Le partage, très fréquent parmi les Touaregs, entre le mariage arrangé (lié à la sphère familiale ou à l'intérêt économique) et la violence étourdissante de tara-n Iblis (litt. « l'amour du diable », confiné à la sphère extraconjugale et lié à l'action des esprits malveillants) 
engendre de nombreuses relations clandestines, qui sont souvent tolérées (dans le cas de l'homme ainsi que de son épouse), à condition qu'elles soient discrètes ${ }^{10}$.

Ce poème permettra aussi de saisir le statut des femmes nobles à l'époque du Père de Foucauld. Dans ce poème, Lalla oult Illi répond à son mari Etteiioub qui lui avait fait savoir être embarrassé par les rumeurs circulant autour de sa relation, courtoise ou adultère, on ne sait, avec le jeune Mousa agg Amastan, destiné à devenir amenokal (chef) des Kel Ahaggar. Dans ce contexte, il n'est pas insolite que les jeunes hommes aient des relations avec des femmes mariées, du même âge qu'eux ou plus mûres.

\section{Enna âs i Etțeiioub : aba ti k! Egrou}

Dis à Attayoub [Etteiioub] : meure ton père ! Écoute bien

Mousa ăgg Ămastan out till ad iouiâ

Mousa agg Amastan n'a pas pareil

Abarad anderren inhegg $\dot{g}$ t tmoulla

Homme tout jeune, il est fait pour les baisers

Edag oua iră dar i étt igrou

La place qu'il voudra dans mon cœur l'aura [litt. « le lieu qu'il a voulu dans moi il le trouvera $\gg$ ]

\section{Lalla oult Illi ent a ten innën}

C'est Lalla oult Illi qui le dit ${ }^{11}$.

Nous avons des poèmes de critique, comme celui de Gagga oult Ăgg (de Foucauld, 1925-1930, p. 69) contre les Kel Tăzălet qui ont manqué de considération à son égard en ne lui offrant pas le repas de l'hospitalité, ou contre son frère qui a voyagé dans l'Adghagh sans lui proposer de partir avec lui.

Le poème narquois qui suit est dû à Sassa oult Oukachcha, née en 1855 (de Foucauld, 1925-1930). Elle ironise sur les comportements des jeunes hommes

10. De nos jours, chez les jeunes femmes touarègues élevées en contexte urbain et fortement exposées à la télévision, des crises de jalousie, imitant les hérö̈nes romantiques des telenovelas latino-américaines, éclatent avec une fréquence redoutable. Ces nouvelles attitudes, jugées déplacées dans le cadre d'un mariage de famille, sont à l'origine de nombreux divorces.

11. Charles de Foucauld, 1925-1930, p. 69. 
qui ne participent plus, élégamment parés, aux réunions galantes. Elle souligne perfidement que leur absence n'est pas motivée par une mort honorable au combat mais par l'épuisement physique dû à la sécheresse qui les marquera à jamais, aux yeux des jeunes femmes, comme des lâches :

Mess-iner, eddèreṛ ad ilkâm

Mon Dieu, puissé-je vivre jusqu'à ce qu'il m'arrive

Nehânnei anĝ̀i tela tetrâm

De voir l'eau de pluie ruisseler sur la terre

\section{Teḍdân tela tâieouân teg்ğân}

Les troupeaux descendre les cours de vallées, paître, se rassasier, s'accroupir repus.

ğânnin "ho-hoo", nekkaniḍ nessân

Et les hommes répéter « ho-hoo », en cadence pour accompagner le violon

Imarder iğ ezzemân izrân

Maintenant c'est le temps de la tristesse et du découragement

Echchchen et tkerbeït ouḍan

Les mousselines blanches et les ceintures de laine rouge sont pliées

Mess issen iekîn aba t ensân

Leur maître est mort, elles reposent

ğânnin: aoua arsi a tt i inrân

On dit : c'est la faim qui l'a tué

ourğir tăzouli, out till ar igân

Ce n'est pas l'épée, car il est resté accroupi dans sa tente.

\section{Deux louanges (temmal) d'A.}

Revenons au festival d'Iférouane. Son but était d'exposer la « culture » touarègue. Il était pris dans des enjeux patrimoniaux, des enjeux politiques et de définition identitaire dans une situation préconflictuelle. Un travail d'explicitation et d'institutionnalisation de la culture et de l'identité touarègue était en jeu, dans un 
contexte globalisé, où l'institution locale, régionale ou nationale se projetait sur une scène qui dépassait la population concernée, causant des tensions. Le festival posait un problème majeur en termes d'explicitation : qu'est-ce que la «culture » touarègue ? Qu'est-ce que la poésie touarègue ? Qui en sont les véritables acteurs ? Comment patrimonialiser le « secret »?

Lors du festival de 2006, trois poétesses furent admises à la compétition poétique après des échanges avec les touristes européens ${ }^{12}$, auxquels il m'arriva de participer, et qui regrettaient une absence féminine sur scène. Et c'est même une femme, A., qui remporta la victoire, avec un répertoire politiquement correct, faisant usage de la lyrique amoureuse, ainsi que de louanges, adressées notamment à un ministre présent au festival et au maire de sa commune rurale. Cette poétesse, d'origine servile et mère célibataire d'un enfant conçu avec un touriste, était, du fait de sa réputation compromise, la candidate idéale pour s'exhiber en public. Cette poétesse « touristique » permettait de répondre aux attentes divergentes des acteurs, les puissantes agences de tourisme et leur clientèle, les tenants d'un Islam rigoriste et les autorités politiques nationales et régionales, adulés dans ses poèmes. Dans un but comparatif, je présenterai deux poèmes d'A., essentiellement des louanges (temmal).

Le premier poème s'ouvre sur une invocation au forgeron Moussa pour qu'il se fasse l'ambassadeur de son message amoureux dans des contrées éloignées, où les jeunes filles ont la fraicheur de la végétation à la saison pluvieuse. Suit la description de l'homme aimé, richement paré et capable de subvenir avec largesse à ses besoins matériels. Le poème se termine avec une louange au chameau Awragh qui effectuera ce voyage, équipé de riches accessoires et d'une belle selle.

Enăd-in Mussa

Moussa, mon forgeron,

wăs yămăra šăhi

À qui j'offre du thé,

wă yadallăgan tyadba

Qui décore les lits,

Zăhaz-du tămăra-năk

Approche ta monture,

12. La position des touristes prônait l'égalité homme-femme et l'empowerment féminin. 
CAHIERS DE LITTÉRATURE ORALE

124 Paroles publiques, paroles confidentielles $-\mathrm{n}^{\circ}$ 77-78

šzwad-in ămăzal-in

Transmets mon message,

Făsufan wăr ămôs kăya

Léger, qui n'est pas un [lourd] bagage

yur itărtar on Tăma

Aux vallons de Tămma

ăhanen tăssa on yonžar

Au cœur des Ighanjar

Dež-ăzyămar ss-ənig

De là, il se dirigera vers l'Est,

Dež-aqqal ifăzran

De là, il retournera dans les vallées,

de kănən əmzuzzar

Qui se succèdent l'une l'autre,

tyibussay

Quelle beauté !

maday tyikăd tăgan fôdan

Comme la lancée des dromadaires,

Mina tăzawzăw ad tărwaq tăganat kăska

Mina, une fusion du vert et du jaune ${ }^{13}$,

Ummă ăkassa on tormăn

Oumma le pâturage de l'ouest ${ }^{14}$

wă ger tyižiră-wen

13. Elle énumère pendant quelques vers les clientes du forgeron, en lui demandant de faire du bon travail pour elles.

14. Il est fréquent de louer une femme en comparant son teint à la couleur de l'herbe, ce que la chanteuse avait déjà fait au vers précédent. 
Entre les hangars là-bas,

wă zayăran y-itrăn

Qu'entourent les étoiles,

Tadrar tămer fell-as

D'un climat doux,

yăbda tadêlăq-qat

Rhabda, garde-la,

dăs tăboda s-omssal

Glorifie-la avec des colorants,

wan d-anzanen zagăr

Qui s'achètent chèrement,

de tan tige behu

Si tu as des doutes,

yur-am tige alyařš

Pour elle, fais un travail bâclé,

$\breve{A d-a ̆ d a g a ~ i n i g ~ w a ̆ y ~ a d-a ̆ f r o n a y ~ e l i l l i ~}$

Je partirais vers l'Est choisir un homme noble,

wă yənagădan təndig

Qui s'enturbanne avec un voile

tondăd-ewărwăr on tyiswat

Avec un large indigo sans limite

wă wăr nalla găḍda yanyal dis ălwărdi

Sans limite,

yəzməzlig tugan yonyal dis ălwărdi

Il porte une gandoura sur laquelle il répand du parfum oney-as Tămălawlaw

Je lui dis, la Brillante, 
126 Paroles publiques, paroles confidentielles $-\mathrm{n}^{\circ}$ 77-78

săglu tyadărka

Envoie le festin,

ku dăr tăwed našitu ăkăla

S'il ne suffit pas, nous l'augmentons,

fel măn năm wă day tyamdiwane

Pour toi et celui avec qui je cause,

yagi betu wăla taweqat

Sa parole restera dans ma mémoire,

Ehan $n$ tamtat yăxarăm-am-tu

La tente féminine, il la frappe d'interdit dans ton intention [signifie probablement : il te la réserve, il l'interdit à quiconque autre que toi].

derănan năm yzyabal-ăm tan

Tes rêves, il te les réalisera,

yal-ifôdan ăbiyăran tan

Il a des chameaux, il s'en occupe,

năk tay betu ătefay-am tat

Je maintiens pour toi [toujours l'interlocutrice imaginaire, ou la poétesse elle-même] ma parole,

tyidwa n-ăwray yadwan tădăra

Louange à Awragh qui arrive à la nuit tombée à Tadara,

wăla buḍda wăla təwequ

Sans crainte, je ne l'oublierai pas,

yal izăwwan ăbarbaran-tu

Il a des cordes, qui l'enserrent,

tarək tămzak.

Et la selle tamzak [selle particulière à la région d'Agadez].

Le deuxième poème commence avec une invocation aux étoiles et à Orion, suivi d'une louange à Hadi, une jeune fille d'une beauté lumineuse et d'une 
honorabilité irréprochable. Elle écoute les conseils et se prépare sagement au mariage en mangeant de la bouille de mil et de lait qui lui assurera une prise de poids, appréciée dans ce contexte. Elle n'aura pour mari ni un akafar (un étranger mécréant, par exemple un Occidental) ni un riche parvenu dont la maison est telle une prison, surveillée par des soldats et entourée de fil de fer barbelé.

Au contraire, un homme noble et patient l'épousera et lui assurera une maison adornée de tapis d'Alger et d'une étincelante vaissellerie américaine, en cuivre et en inox. La deuxième partie du poème est constituée par une longue louange à Rhissa (probablement Rhissa ag Boula, ministre du tourisme présent le jour du concours). On dépeint Rhissa en héros et en croyant, qui n'a pas son pareil en Arabie et qui, parmi les oiseaux, ressemble à un épervier blanc. Toute la communauté est prête à le suivre, à lui balayer des nattes pour qu'il s'assoie, parce qu'il apportera de l'or et réalisera les rêves de bien-être économique. Suit une invocation aux femmes élégantes de Gougaram et une louange au maire de sa commune, Adam, brillant comme Orion et élancé comme un haut palmier qui surpasse les autres. Le poème se termine par une invitation aux femmes de sa contrée à bien laver leur or pour faire bonne figure face aux femmes de Gougaram, qui les attendent à Iférouane et ressemblent à des gazelles.

Itrăn amănar anta as tăkăsa isəm

Parmi les étoiles, c'est Orion que je remémore,

Xadi tabarart-in tôbadăt todirka

Hadi, ma fille, suit son régime de mil pilé mêlé d'eau,

taslăt-ya taraqem ad tălkas n-aliwa

Elle écoute les conseils [sur] la calebasse de bouillie sucrée,

trqanăt tazôka tuway ak ayôra

Elle porte un bracelet, et veille chaque matin [elle reçoit les visites nocturnes des hommes qui la courtisent],

Ažil wăn tăbăde tămbay wăn tămale

Le jour du rassemblement, elle apparaît comme la lumière de la lune,

War anabrer agnăw, tasxa fel ažina

Que ne cache le nuage, elle est plus pure qu'une pluie, nassalil-tăt agna wăn kura qătemăn 
128 Paroles publiques, paroles confidentielles $-\mathrm{n}^{\circ}$ 77-78

Nous lui portons le boubou indigo et des chaussures,

Lăwni anten tyayiswen ațtagen răwăyan

Une cérémonie riche en couleurs,

Išilăn $n$ akal-net adoy talăžanăn

C'est sa contrée qu'ils envient, wăn amăylol wăyalan tamina

Elle est éternelle et respectable, yaクu kud tămowăy s-ayiwăn n-akafar

Et qui dira qu'elle ne sera transportée ni à la maison du mécréant, wăla yən emalyi yəknan as samanti

Ni à celle du riche, cimentée

yaylăytu essasar

Encerclée par un fil de fer barbelé, yagardatu sôži wăn ešku n-azăngo

Gardée par un militaire, ennemi et esclave, yoyadăn sagari yasasu ark taba

Qui fume la cigarette [litt. qui boit les mauvais tabac],

Xadi tăbărt-in taqablăt tămert-net

Hadi ma fille, fille de son temps, ehan wâ totagaz yokras akătăbba La case dans laquelle elle loge est merveilleuse ahăn damasatăn, ibroyăn tăzalge

On y trouve des belles couvertures d'Algérie [litt. du Nord], akiyăn d-emižinet

L'un après l'autre, Yahe elili yalwăn as tezeyder

S’y trouve un noble armé de patience, 
taga taniqes ta-dafir almoz

Se passent des causeries après le crépuscule,

emastăn tămažgôl war tyanmănžay allă

təylăy-tu tematyat ažasitrom ehad

L'encerclent des gens jusqu'au milieu de la nuit,

yožil arăgăyga aseten n-imorka

En abondance, du thé vert dans des assiettes américaines,

War tuwet Frănsa

Que la France n'a jamais conçues,

Taza wan takirmay Išn inôx

Neuf ustensiles en verre, deux en inox,

Yaben atărăya yijwaden-di-dăroy

Parmi eux un récipient en cuivre.

mani izubun medan

Où est l'honneur des hommes,

tegatew an temet

Le pilier de la communauté,

anămăgăr yasa

Le guerrier Rhissa,

Jasa yasăn dəy allăbu

Rhissa croit en Dieu,

Wa yatafän man-ən

Qui détient mon âme

Yxxkăm amazal-ən

Gouverne ma mission,

Yasan-day-i albumma

Qui sait en moi, le miracle, 


\section{CAHIERS DE LITTÉRATURE ORALE}

130 Paroles publiques, paroles confidentielles $-\mathrm{n}^{\circ}$ 77-78

yasa tyašenen-nak dəy tagăma iyuras

Rhissa, en ton honneur, je puise des puits profonds,

yəsa tyəšenen-nak as tăkkala almuxal

Rhissa, en ton honneur, je me mets en péril,

yəsa tyəšenen-nak as lăkkăda manən

Rhissa, en ton honneur, je suis mes traces,

ondazal as takos

Hier soir,

nagla nasikabăr

Nous marchions en glorifiant Dieu,

kompănyi žə-nnəka

En direction d'une compagnie,

kompănyi wăn farwan

La compagnie d'Iférouane,

nagla sayat $n$-alxer

Nous marchons d'une bonne chance,

Samăl za wăn yasa

Le portrait de Rhissa,

War nonay day aytăd̆ăn

Nous ne le voyons pas chez les hommes,

Wala day arabăn won asoyudiya

Même chez les Arabes de l'Arabie,

Naqqal-tu sar igḍd wan war nola abăkad

Nous l'avons cherché chez les oiseaux

Yasaktatya alhuḍhuḍ zney găgidaran

Qui n'ont point de pêchés, Il me rappela la huppe, je me suis dit, ...

yəsa bašar măllan war təniy azalom 
Rhissa, l'épervier blanc qui n'aperçoit pas un malveillant

Wan ark mazalän

Qui fait des péchés

Wa yatanney aliman

Celui qui voit un imam

Wa žzylayan makkat

En pèlerinage à la Mecque,

Wa yatăynay aliman yəkkan tamazgada

Qui aperçoit un imam en direction de la mosquée,

yosa nasəkabar a-năgruw anăy-nak

Rhissa, nous glorifions Dieu, pour vous voir,

Won Gugaram medan nəră d-ahan esuk

Les hommes de Gougaram, nous les voulons au marché,

Naga ațtima a-năgruw wă-yagan kamăn yəsa

Nous espérons en avoir un comme Rhissa,

Wala ż-amos gurgu taburuk aš-agla

Même s'il est un handicapé qui marche avec un bâton,

Nazirgaz-ən tyamnas tăn-du nazənzu

Nous enverrons des chamelles pour l'acheter,

Dan-axbilăn deran dan-agan uray

Pour qu'il nous réalise les rêves, pour qu' il nous donne de l'or,

Natiytiy nagu esaber dat-as

Nous dressons [et] installons un paravent devant lui,

Nəfarad-as tyazgar žatayas ellili

Nous balayons [les nattes] pour lui, un noble galant s'avance,

As-amdanet tyaftay

À la fin des causeries, 
132 Paroles publiques, paroles confidentielles $-\mathrm{n}^{\circ}$ 77-78

\section{Tyən Gugaram šatma toglămat ezənbiz}

Mes sœurs de Gougaram, vous ne méritez pas l'indifférence,

ešikil ən par Bibi nakku ažagan sankad

La troupe de Bibi, qui me fascina,

yažilaylay uray, tiga ilašăn

Remplie d'or, d'indigo,

par Bibi ezayan tufat

Bibi, la lumière de l'aurore

wa žabdayan Tamgak s-alqablat on Makăt

Qui depuis la Kabba de la Mecque éclaire Tamgak,

Xawa tala Kallo yaxraki samal-net

Hawa a Kallo dont je ne sais à qui la comparer.

Merăn wan akalon adăm d-ilxukuman

Les maires de ma contrée, Adam parmi les autorités,

Adam d-alxukuman amanar d-ažina

Adam parmi les autorités, l'Orion dans l'univers,

Adam way tegətew təkbalat taffăla

Cet Adam-ci est le pilier qui soutient le plafond,

Aš-abdad day meddan yabdid wan tanala

Dès qu'il se lève parmi les hommes,

bagzan dalyumuran

Il est visible telle une plante oasienne,

Tăzăwzăwn-elam-net tofa essăm n-ayora

Son teint est mieux qu'un éclair matinal

Yatagan day tyawgas

Qui se fait dans les champs,

Yəzənzar abora 
Qui fait pousser du maïs,

Yəkraz anasera

Il est cultivé avec bonheur,

yur tămer n-ažina

À la saison des pluies.

Yoxdam atefin yakusan war alyen

Que travaille un haoussa dur à la tâche, qui n'est pas paresseux

šatma ayradnet malăy-akmăt

Mes sœurs, je m'adresse à vous toutes,

Uray nakmăt yonset day aman

Votre or, lavez-le avec de l'eau

Soklan war-kulu žardan

Avec du savon, ne le laissez pas malpropre.

Tyan Gugaram aqqalnat-ăna

Celles de Gougaram, nous attendent,

Aš-əga onki war təffa sămal

Et je ne donne aucune autre comparaison,

Kudba adigalšet tufat s-ayora

Jusqu'à demain matin

Adänbornat onken tyoneren

Elles apparaîtront telles des gazelles.

Ce poème, constitué par des louanges de différents individus ou groupes de personnes, ne présente pas d'excessive difficulté de compréhension parce qu'il n'est pas aussi métaphorique que celui de $T$, que nous présenterons dans le prochain paragraphe, tellement ses propos sont affichés d'une manière explicite. La poétesse s'adresse aux autorités politiques, influentes au niveau national et local, et véhicule la nouvelle idéologie de l'ăzăman, de l'époque où la circulation de biens de prestiges en provenance de pays lointains (Algérie, France, Amérique) produit des désirs exacerbés de nouveaux objets et de l'or (uregh) à profusion. Ainsi le chanteur Bambino, un afaghis de la région d'Agadez, nous présente avec 
134 Paroles publiques, paroles confidentielles $-\mathrm{n}^{\circ}$ 77-78

amertume et appréhension la soif de biens matériels qui conduit au changement de la conception de l'amour, dans la chanson « Ăzzăman n tilyaden » (l'époque des jeunes filles).

Awen ăzzăman

Cette époque

ăzzăman $n$ tilyaden

Est l'époque des jeunes filles

Tarha-nasnat

Leur manière d'aimer

Tiglat isgabayyin

A complètement changé

\section{Yalla iwat ayitma}

Que Dieu vous protège mes frères

Yoffa anulu gabba

Mieux vaut veiller attentivement

Fel tilyaden-nanagh

Sur les jeunes filles de chez nous

Tin $n$ anada $n a ̈ z z a ̈ m a n$

Celles de nos jours.

\section{Poème de T.}

Pour terminer, je présenterai le poème de $\mathrm{T}$. au lecteur, qui pourra le comparer aux anciens poèmes féminins de l'Ahaggar et de l'Adghagh et apprécier la différence avec les louanges de sa contemporaine $\mathrm{A}$.

T. commence son poème par une invocation à Dieu qui lui a livré des étrangers belliqueux sans monture et sans outre pour boire. Le poème se poursuit avec une description du narrateur qui a été créé avec toutes les qualités, à l'image de l'ange et d'une tradition guerrière qui suscite l'admiration. Suit la description ironique de leur chef qui rend visite au narrateur avant l'aube et sans prier. Si, dans les louanges d'A., Rhissa était comparé à un épervier blanc et Adam à un haut palmier, le chef géant ressemble dans le poème de $\mathrm{T}$. à un gros arbre, à une large natte et 
à une énorme portière. Ces expressions suscitent une hilarité certaine auprès des auditeurs touaregs pour différentes raisons en relation avec le système de valeurs éthiques et esthétiques.

Dans cette société, la légèreté et la simplicité sont les caractères propres à la beauté, tandis que la laideur se manifeste en tout ce qui est lourd, voyant et trop chargé. Un homme gros, en plus, sera perçu comme faible d'un point de vue moral, la gourmandise renvoyant à une attitude paresseuse, peu adaptée à un guerrier qui se caractérise par la sveltesse de ses mouvements mis en relation avec la rapidité de ses capacités cognitives.

Ensuite, un homme honorable ne devrait jamais manger à sa faim mais laisser son épouse et ses enfants se nourrir davantage que lui. Pour cela, il s'éloigne poliment d'un demi-mètre de la natte où sa famille consomme encore le repas. Les hommes touaregs se font un point d'honneur de protéger les individus plus faibles qu'eux, les qualités morales et la réussite d'un homme sont jugées sur la base des rondeurs de sa femme et de la largesse avec laquelle il habille sa mère.

Si une femme grassouillette est considérée comme attirante dans la majorité des groupes touaregs, un homme gros est souvent l'objet de moqueries, car on lui reproche sa gourmandise, son égoïsme et sa paresse. Le style de vie et l'alimentation des sédentaires favorisant la prise de poids, les hommes touaregs fonctionnaires en ville sont souvent comparés par les nomades à des « gros Haoussas » ou aux matrones touarègues qui, mères de plusieurs enfants, sont souvent obèses.

Le chef étranger décrit par T. n'est pas seulement un mécréant et un paresseux (physiquement et mentalement) mais aussi, avec une tăngălt (parole métaphorique et nuancée) un corrompu. Son corps est comparé aux « plâtres » qui revêtent les mains et les pieds des femmes, lorsqu'elles passent de nombreuses heures dans l'immobilité pour que la peau s'imprègne des dessins au henné.

Dans les poèmes, l'argent que l'on veut dissimuler est emballé dans les plâtres. Il s'agit ici d'une critique abrasive où l'appropriation illégale d'argent et la corruption du chef sont dénoncées, en le ridiculisant à travers l'évocation des femmes oisives et obligées à l'immobilisme. Le poème se termine avec une dernière invocation à Dieu et avec l'évocation du sabre vengeur sur lequel la foule des croyants et des mécréants (un clin d'œil, peut-être, aux touristes présents au festival), rassemblée par la volonté divine, concentre son regard, en proférant des invocations à Dieu.

Après ces mots agressifs et allusifs, susceptibles d'enflammer une foule qui réagit déjà avec des cris véhéments émis par les hommes et des youyous d'encouragement de la part des femmes, le microphone est soustrait à T. qui est poussée hors de la scène. 
Wă di-yalan ontay ă-yalăn tănăyyă,

Celui qui me possède [Dieu], c'est lui qui a la toute-puissance, wă di yaffan əmăqqarăža, Lui qui m’a livré des belliqueux, d-aglănen dăy ăkal wă yăgugan, Venus d'une contrée lointaine, waliyăn wăr alăy tămăra, Aucun d'entre eux n'a de monture, wăr alăy ibyay dăy zšwa, Ni d'outres d'eau pour boire wăn ăngălôz a-šiyzkna, L'ange qui m'a forgé. ăgôz wăn băgzan a-war-di-yagga,

Un jarret de Bagzan, ce n'est pas ce qu'il m'a fait : il ne m'a pas donné un jarret comme en ont les chevaux du mont Bagzan [race de chevaux réputée pour sa rapidité] ${ }^{15}$.

yəgi-du dăgh ăgna ən tăkôba,

Il m'a fait dans la tradition de l'épée ${ }^{16}$, Er tătyazlagan yakôday wala žamôs ărăbayya,

Celui qui la [l'épée] porte au côté est poussé à la curiosité [désireux de voir ce qui arrive et non de fuir], même s'il n'est qu'un adolescent Yazgăran war-ăha waliyăt,

Passant innocemment son chemin, onner-as tăkănu ar ăgna,

Je lui dis, qu'il réalise son choix.

15. Ici, le narrateur énonce qu'il n'est pas un lâche, prêt à fuir en cas de danger. 16. Le narrateur affirme son statut de noble. 
wăn ămyar năssan ătăžamma,

Leur chef est gros et gras [Atazamma est un idéophone qui veut dire gros],

As-дžăymăs arwă wăr ăffô,

Il me visita dès l'aube,

yəžalăyžălay dăy tănăyă,

Marchant difficilement, [parce qu'il ne voit pas bien dans l'obscurité de l'aube],

wăr ămmmud wăr okațtăt yălla,

Sans prier car ignorant Dieu,

eyăf-net tăbuk a-dar ôla,

Sa tête est blanche comme une fleur [le narrateur sait presque qu'il a affaire à un homme d'un certain âge, mais dans l'obscurité il doute encore],

Ad-asmătkala ăket assaka,

Je le regarde : quel gros chamelon! [litt. quantité chamelon, ăket, quantité, sert souvent à introduire des exclamations familières ; la répétition du mot ne peut que déclencher les rires],

An-asmănômăn ăket tădăda

De loin je le scrute : quelle grosse charge de chameau !

Ann-aswărăwăr ăket ăssăla

Je l'inspecte intégralement : quelle grosse natte ! [aswărăwăr, tout comme asmănômăn au vers précédent sont des mots que la poétesse a fabriqués en redoublant la racine ; le procédé ajoute à la tonalité burlesque du passage],

ăket tăyăssa ățtănăkfos azrəf s-anolla,

Quel gros corps ! Comme de l'argent emballé du henné.

ayreqqa emăli wăn tănăyya,

J'invoque Dieu, le Tout-Puissant,

wă-di dəxlăkan 
138 Paroles publiques, paroles confidentielles $-\mathrm{n}^{\circ} 77-78$

Qui m’a créé.

tădaw tăxlikt er tăt yalan

Une foule s'est rassemblée, quiconque en faisant partie

ta dăy an nasliman a-kad tăn kufär

Qu'il s'agisse des musulmans et même des Occidentaux [kufär signifie d'abord « mécréants » et sert usuellement à désigner les Occidentaux; il ne semble pas ici avoir son sens premier : les kufär en question font des invocations pieuses !]

de-tu tillay tănăyă tassăggăd

Où que la vue porte, la foule regarde

tăzyayt tay totătet tănăyă

Le sabre envié par les vivants,

de-tyilla imi yašewăl

Chaque bouche, quelle qu'elle soit, bavarde,

de-tu tillay tănăyă totățtăr

Où que la vue porte, la foule profère des invocations.

Quarante jours après le festival, la caserne d'Iférouane fut attaquée par les rebelles du MNJ, l'oasis entièrement minée, et la population, fuyant en masse la zone du conflit, se réfugia dans le chef-lieu, Agadez, en état de siège militaire.

\section{Bibliographie}

Appadurai Arjun, 2001 [1996], Après le colonialisme : les conséquences culturelles de la globalisation, Paris : Éditions Payot \& Rivages.

BERnus Edmond, 1972, «Incongruités et Mauvaises paroles touarègues », Journal des Africanistes, no 42-1, p. 89-94.

BERnUs Edmond, 1989, « Paroles convenues : mots et jeux de mots touaregs », in Graines de paroles, puissance du verbe et traditions orales, Paris : CNRS Éditions, p. 79-89. 
Casajus Dominique, 1981, « la Tente et le Campement chez les Kel Ferwan », Revue de l'Occident musulman et de la Méditerranée, nº 32, p. 53-70.

Casajus Dominique, 1987, « Parole retenue et Parole dangereuse chez les Touaregs Kel Ferwan », Journal des Africanistes, n 57-1-2, p. 97-107.

Casajus Dominique, 2000, « la Parole pénombreuse des hommes voilés », in Bertrand Masquelier, Jean-Louis Siran (dir.), Pour une anthropologie de l'interlocution, Rhétoriques du quotidien, Paris : l'Harmattan, p. 313-335.

Claudot-Hawad Hélène, 1993, « Femmes touarègues et Pouvoir politique », in les Touaregs : portrait en fragments, Aix en Provence : Éditions Édisud, p. 119-131.

De Foucauld Charles, 1925-1930, Poésies touarègues (dialecte de l'Ahaggar), 2 vol., Paris : Leroux.

DE Foucauld Charles, 2005 [1951], Dictionnaire touareg-français, 2 vol., Paris : l'Harmattan.

Doluet Anne, 2007, « l'Enquête en “situation ethnographique” ou l'exercice nécessaire de la réflexivité », in Olivier Leservoisier, Laurent Vidal (dir.), l'Anthropologie face à ses objets : nouveaux contextes ethnographiques, Paris : Édition des Archives Contemporaines, p. 205-222.

Dragani Amalia, 2012, «Interno tuareg. Etnografia dei poeti nomadi del Niger», Roma: Ed. Aracne, « Scienze sociali ».

Elmeki Mohamed, 1979, la Satire dans la poésie tamasheq (Kidal), mémoire de l'ENS, Bamako.

Fabre Daniel, Jamin Jean, 2010, « Auto-biographie, Ethno-biographie », l'Homme, no 195-196.

IsnarT Cyril, 2012, les Patrimonialisations ordinaires : essai d'images ethnographiées, Ethnographiques.org, $\mathrm{n}^{\circ} 24$. 
JAY Marie, 1999, « Rire entre femmes : parenté, alliance et sexualité (les Kinni Touaregs d'Abbéché - Tchad) », in Éliane Daphy et Diana ReY-Hulman (dir.) avec la collaboration de Micheline Lebarbier, Paroles à rire, Paris : Publications Langues'O/INALCO.

Lassibille Mahalia, 2006, « les Danses woDaaBe entre spectacles touristiques et scènes internationales: les coulisses d'une migration chorégraphique $\gg$, in Anne Doguet et Sara Le Ménestrel (dir.), Autrepart, n 40, p. 113-129.

Pandolfi Paul, 2001, « les Touaregs et Nous : une relation triangulaire ? », Ethnologies comparées, $\mathrm{n}^{\circ}$ 2. http://recherche.univ-montp3.fr/cerce/revue. htm

Prasse Karl, 2003, Dictionnaire touareg-français, 2 vol., Copenhague : Museum Tusculanum.

Rasmussen Susan Jane, 2004a, "Reflections on Witchcraft: Danger, and Modernity among the Tuareg", Africa, vol. 74, n 3, p. 315-34.

Rasmussen Susan Jane, 2004b, “'These are Dirty Times!' Transformations of Gendered Spaces and Islamic Ritual Protection in Tuareg Herbalists' and Marabouts Al Baraka Blessing Powers", Journal of Ritual Studies, $\mathrm{n}^{\circ} 18-2$, p. 43-60.

TAuzin Aline, 2007, « Femmes, Musique et Islam : de l'interdit à la scène », Clio, Femmes, Genre, Histoire, no 25, p. 133-153.

Résumé : À partir d'un cas ethnographique - un concours de poésie touarègue au sein d'un festival au nord du Niger - cet article aborde la relation entre l'espace public et religieux, le genre et les normes, contestés, d'usage de la parole à la veille de la rébellion touarègue de 2007-2009. L'exclusion des femmes du concours poétique suscita différentes réactions de la part des intéressées. Les poétesses les plus jeunes furent marginalisées en raison du contenu politique de leur production. Une poétesse seulement, T., tirant légitimité de son aînesse, appuyée en cela par une partie du public et par les poètes, s'autorisa à saboter le concours en s'invitant à tout moment sur la scène, en volant le microphone et en déclamant ses tišiway (poèmes). Cet article présente un « poème de critique » récité par T. lors du concours. Après avoir abordé brièvement le champ sémantique du 
rire dans la société touarègue, des exemples de la production satirique féminine sont offerts pour contextualiser le poème de T. à l'intérieur d'un genre poétique touareg, l'abayak (littéralement « critique », satire ou invective). En 2006, après des négociations avec les touristes, des femmes furent admises au concours. L'une d'elles, A., remporta la victoire. Deux de ses poèmes rentrent dans le genre de la louange (temmal) notamment aux autorités politiques présentes. L'article les compare avec la critique voilée mais abrasive de T. et des poétesses « engagées », qui demeurèrent exclues du concours.

Mots clés : poésie orale, tourisme, Touaregs (Sahara), patrimoine immatériel et création, conflits armés, pouvoir, agentivité, genre

Abstract: Starting with an ethnographic case - a Tuareg poetry contest in a festival in Northern Niger - this paper discusses the relationship between public and religious spheres, and between gender and disputed norms of speech, before the Tuareg rebellion of 2007-2009. The exclusion of women from a poetry competition aroused different reactions on behalf of the interested women. The younger she-poets, famous authors during the rebellion of the 1990s, were marginalized in light of the political content of their production. Only a senior poetess, T., who derived legitimacy from her birthright, supported in this by some of the audience and some poets, allowed herself to sabotage the competition by intervening on frequent occasions, stealing the microphone to recite her tišiway (poems). This article presents a "critical poem" that is recited by $T$. during the competition. The paper focuses briefly on the semantic field of laughter in Tuareg society, and examples offemale satirical productions contextualize T.'s poem within a literary Tuareg genre, the abayak (literally "critical satire" or invective). In 2006, women were admitted to the competition after negotiations with tourists. Then A., a woman, won first prize. Two of A.'s praise poems (temmal) are compared with T.'s veiled but abrasive critique.

Keywords: Oral Poetry, Tourism, Tuaregs (Sahara), Intangible Heritage and Creation, Armed Conflicts, Power, Agency, Gender 